\title{
Modelling of systemic uptake of agrochemicals after dermal exposure; effects of formulation, application and exposure scenarios
}

\author{
Jacob Krüse · Christel W. E. Verberk
}

Published online: 5 September 2007

(C) Springer Science+Business Media, LLC 2007

\section{Introduction}

Dermal exposure plays an important role in the risk assessment of agrochemicals. A number of dermal exposure models are available to estimate to external amount of material deposited on the skin during various activities. Since the skin forms a barrier to the uptake of chemicals, only part of the material on the skin will actually be absorbed into the body. In the absence of experimental data usually default values are used for the degree of dermal permeation (10 or $100 \%$ of the external dermal dose). These values are related to the physical chemical characteristics of the compound (De Heer et al., 1999). On the other hand, mechanistically based mathematical models that describe the dermal absorption and incorporate compound specific information can be used to estimate the dermal permeation of a compound under specific occupationally relevant exposure conditions. In this paper such a model is introduced and used to illustrate the effects of exposure scenarios (e.g. repeated, multi-day exposures). Furthermore the possibilities of the model are discussed with respect to the assessment of the effects of various formulation types (water solutions, dry powders, and granules) of agrochemicals on the dermal uptake. Also the influence of other factors on dermal uptake, such as the exposed body surface, the volume of the solution applied and the solubility of the compounds and used solvents are discussed.

J. Krüse $(\bowtie) \cdot$ C. W. E. Verberk

ENVIRON Netherlands B.V, Zeisteroever 17, 3704 GB Zeist,

The Netherlands

e-mail: jkruse@environcorp.nl

\section{Methods for dermal risk assessment}

\subsection{General approach}

Dermal risk assessment compares an estimated (or measured) dermal exposure with the acceptable dermal exposure derived from toxicity data of the compound or the plant protection product. Systemic toxicity after dermal exposure may be derived in a direct way from dermal toxicity studies or indirectly from administration via another route (e.g. oral route) combined with a so called route to route extrapolation. In the last approach it is important to assess the percentages of the administered doses that are systemically absorbed via the two routes. In general it is assumed that a fixed fraction of the administered or applied compound will be systemically absorbed via a specific route, and that this fraction will only depend on the physical and (bio)chemical properties of the compound. This may be approximately true for the oral route, but certainly not always for the dermal route. The percentage that is dermally absorbed will depend on various conditions, such as amount applied, dermal area dose, volume applied, physical state (solid, solution, vapor) and vehicle solvent used. These conditions may affect the outcomes of both the direct dermal toxicity experiments and the assessment of the percentage that is dermally absorbed.

\subsection{Estimation of external dermal exposure}

The dermal exposure can either be estimated using generic models or by measurements in field situations. Usually the exposure levels are estimated and only if these are far above the acceptable exposure levels, field measurements are performed. 
Several models for the estimation of dermal exposure have been developed in the past 10-20 years. For the estimation of operator exposure during the application of plant protection products the following models are available: the Dutch model (Van Hemmen 1992), the UK model (POEM; PSD 1986; 1992), the German model (Lundehn et al., 1992), EUROPOEM (AIR 1996) and the Pesticide Handler Exposure Database of the United States and Canada (PHED; Versar Inc., 1992). At present EUROPOEM is still being developed and validated.

The exposure models differ in:

- Derivation of the surrogate exposure values (statistics: geometric mean, 75 th or 90 th percentile).

- Underlying assumptions, i.e. the use of personal protective equipment (PPE).

- The database with exposure data on which the models are based.

In the models a distinction is made between mixing/loading and application of the plant protection product. However, in most cases it is the same person who performs these two activities.

Important factors for the dermal exposure estimations are:

- Inside or outside activities: outside there is influence of weather conditions;

- Duration of task.

- Mixing and loading technique: this can be a closed system, but also manual mixing of the product.

- Application technique: manual spraying with a backpack or other manual equipment or mechanical spraying by tractor mounted equipment. Also automatic spraying systems are available.

- Use of personal protective equipment (PPE), like gloves, impermeable clothes.

- Personal hygiene.

The exposure models yield a potential dermal exposure, expressed as $\mathrm{mg} /$ person/day. This potential dermal exposure is the amount present outside a persons body and on the clothes. In some cases also the actual exposure is estimated by dividing the potential exposure with a certain factor to account for the protection by clothes. Separately, often a specific reduction factor for gloves is used, when appropriate gloves are worn.

It is important to derive a realistic dermal exposure, which can be compared with the acceptable dermal exposure. For this risk assessment the dermal absorption is of importance. When no experimental data are available for dermal absorption, often default values of $10 \%$ or $100 \%$ of the external dermal dose are used, depending on the physical chemical characteristics of the compound (De Heer et al. 1999). These default values are based on a worst case approach and do not take into account the effects of specific conditions of exposure, such as form and level of the applied dose, duration of the exposure and specific (multi-day) exposure scenarios. In this respect mathematical modeling of dermal absorption can play an important role to arrive at a more realistic assessment of the dermally absorbed dose and the resulting risk.

\subsection{Estimation of dermal absorption}

\subsubsection{Structure of the skin barrier}

The skin acts as a barrier to protect the body from external influences, such as toxic compounds that are deposited on the skin. However, this protection is not complete. For a better understanding of the dermal permeation process a brief description of the skin structure is presented here. On the outside of the skin the stratum corneum (SC), a dense lipophilic layer of dead keratonicytes, forms the main skin barrier. The thickness of the stratum corneum ranges from about 10 to $20 \mu \mathrm{m}$. During dermal exposure the outside of the stratum corneum will be in contact with e.g. a solvent in which the permeating compound is dissolved. At the inner side the viable epidermis (VE), a layer of about $100 \mu \mathrm{m}$, is situated. This layer, which is much more hydrophilic and less dense than the stratum corneum, may form a barrier for highly lipophilic compounds (Cleek and Bunge 1993). The viable epidermis is at the inner side in contact with the systemic blood circulation that provides a rapid removal of the material that has passed the skin barrier to the rest of the body, thereby preventing a concentration build up at the inside of the skin barrier ( the so-called sink condition). A schematic representation of the skin during dermal exposure is given in Fig. 1.

\subsubsection{Theoretical aspects of skin absorption}

In its simplest form the dermal permeation through the skin is described by diffusion across a single barrier (in this case the stratum coreum), according to Fick's First Law of Diffusion. The resulting permeation rate or flux $\mathbf{J}$ (rate of mass transported / surface area) is in this case proportional to the concentration difference over the skin: 
Fig. 1 Schematic representation of the skin barrier and the compartments involved in dermal permeation

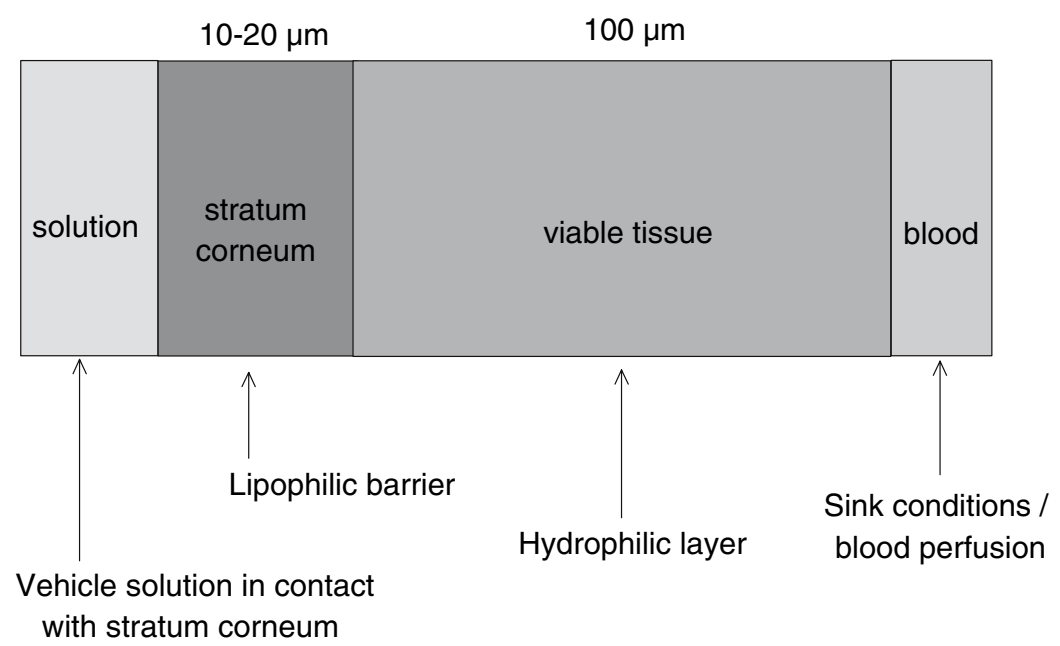

$\mathrm{J}=\mathrm{K}_{\mathrm{p}} \times\left(\mathrm{C}_{\text {applied }}-\mathrm{C}_{\text {inside }}\right)$

$\mathrm{J}=\mathrm{K}_{\mathrm{P}} \times \mathrm{C}_{\text {applied }}$

whereby:

$\mathrm{C}_{\text {applied: }}$ : Concentration in the solution applied on the skin $\left(\mathrm{mg} / \mathrm{cm}^{3}\right)$

$\mathrm{C}_{\text {inside }}$ : Concentration at the inside of the skin barrier $\left(\mathrm{mg} / \mathrm{cm}^{3}\right)$

$\mathrm{K}_{\mathrm{p}}$ : Permeability coefficient $(\mathrm{cm} / \mathrm{h})$

$\mathrm{J}$ : Flux $\left(\mathrm{mg} / \mathrm{h} / \mathrm{cm}^{2}\right)$

This equation is only valid for an equilibrium concentration distribution across the skin. This so-called steady state may only be reached after sufficiently long durations of exposure (depending on the properties of the permeating molecule), provided that the concentration in the applied solution does not decrease during the exposure (so called infinite dose application). In practice steady state conditions are hard to achieve.

If the stratum corneum is the single barrier for skin permeation, the permeability coefficient will depend on the diffusion coefficient $\left(\mathrm{D}_{\mathrm{sc}}\right)$ of the molecule through the barrier, the thickness of the barrier $\left(\mathrm{L}_{\mathrm{sc}}\right)$, and the partition coefficient between the barrier and the vehicle solution on the skin $\mathrm{P}_{\mathrm{sc} / \mathrm{ve}}$, in the following way:

$\mathrm{K}_{\mathrm{p}}=\mathrm{P}_{\mathrm{sc} / \mathrm{ve}} \times \mathrm{D}_{\mathrm{sc}} / \mathrm{L}_{\mathrm{sc}}$

The partition coefficient $\left(\mathrm{P}_{\mathrm{SC} / \mathrm{VE}}\right)$ and the diffusion coefficient $\left(\mathrm{D}_{\mathrm{SC}}\right)$ are depending on the penetrating compound. $\mathrm{P}_{\mathrm{SC} / \mathrm{VE}}$ is related to the lipophilicity $\mathrm{P}_{\mathrm{OW}}$ (octanol water coefficient) and $D_{\mathrm{SC}}$ to the size of the molecule (MW).

When we assume sink conditions at the inside of the skin barrier, due to rapid removal of the compound by blood perfusion, the concentration at the inside $\left(\mathrm{C}_{\text {inside }}\right)$ will be negligible. The flux becomes then:
If the applied concentration remains constant during the exposure, the cumulative amount absorbed will be proportional to the duration of exposure:

$\mathrm{A}_{\mathrm{abs}}=\mathrm{K}_{\mathrm{P}} \times \mathrm{C}_{\mathrm{applied}} \times \mathrm{T}_{\mathrm{exp}}$

whereby:

$\mathrm{T}_{\text {exp }}$ : Duration of exposure

$A_{a b s}$ : Cumulative amount absorbed through the skin into the body

Thus according to equation (4) a graph of the cumulative amount absorbed through the skin into the body $\left(\mathrm{A}_{\mathrm{abs}}\right)$ versus exposure duration $\left(T_{\text {exp }}\right)$ will result in a straight line through the origin. However, as already indicated above, this is only the case if equilibrium across the skin has been attained, and thus not for short exposure duration.

In fact we have to distinguish two processes in the skin absorption process:

1. Absorption from the solution into the skin

2. Absorption through the skin into the body

The cumulative amounts absorbed versus the exposure time for these two processes are represented for a hypothetical case in Fig. 2, together with the absorption according to Fick's first law (Eq. (4)).

The graph shows a rapid increase in the amount absorbed into the skin (curve 1) during the first $6 \mathrm{~h}$, after which a steady state is attained. The amount absorbed through the skin into the body slowly increases in the first few hours of exposure. After establishment of the steady state the three curves will become parallel to each other, meaning that rates of absorption will be equal. It is obvious that simple application of Fick's law results in an overestimation of the 
Fig. 2 Cumulative absorption into the skin (1), through the skin (2) and according to Fick's First Law (3)

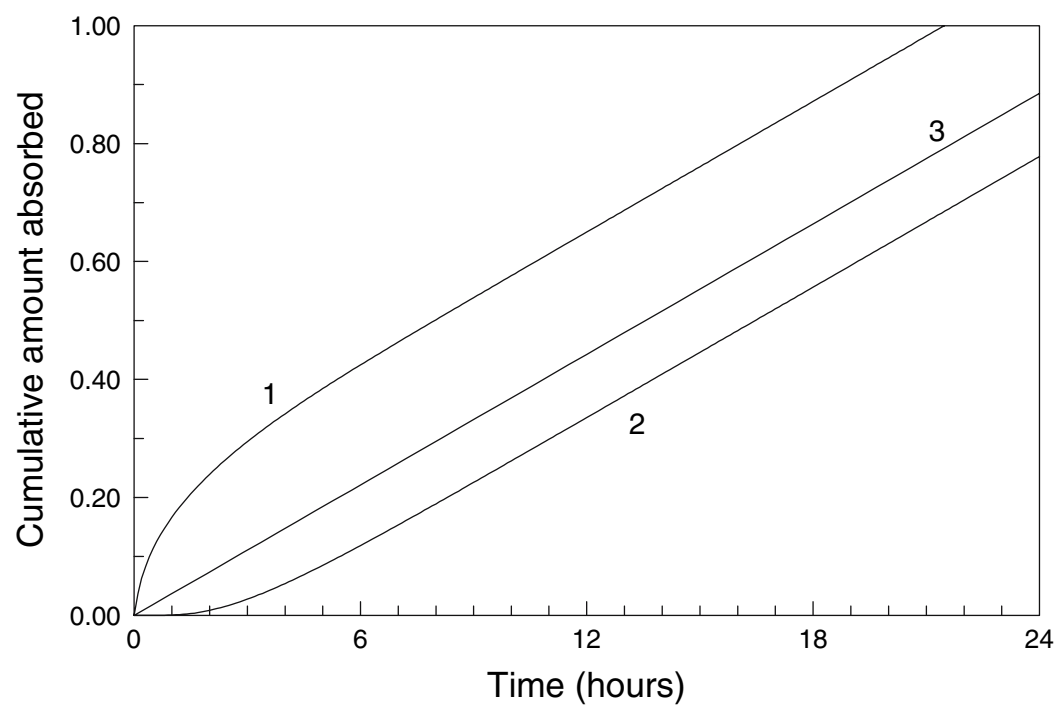

amount systemically absorbed and an underestimation of the amount absorbed into the skin, which is also the amount potentially absorbed into the body after cessation of the exposure.

\subsubsection{Mechanistically based mathematical modeling and simulation of the dermal uptake}

From the curves presented in Fig. 2, it is obvious that a simple application of Fick's law, thereby applying the permeability coefficient does not yield a proper estimate of the dermal absorption, especially for relatively short durations of exposure, when steady state conditions have not been reached yet. For a more reliable approach of the dermal absorption process, a mathematical model was developed in which the physical and chemical characteristics of the skin were incorporated. The skin barrier in the model consists of the stratum corneum and the viable epidermis as shown in Fig. 1. Furthermore, two additional compartments are distinguished, the vehicle compartment containing the dissolved permeant and in contact with the stratum corneum, and the blood compartment which acts as a sink for the permeant that has passed the skin barrier. In the transport of material through the skin barrier both partitioning of material between the vehicle, the stratum corneum and the viable epidermis, and diffusion through the stratum corneum and the viable epidermis are involved.

A schematic representation of the transport through the skin is given in Fig. 3.

The transport between the four compartments is described by a set of mass balance equations. These differential equations are numerically integrated using the
Fig. 3 Transport of material through the skin barrier

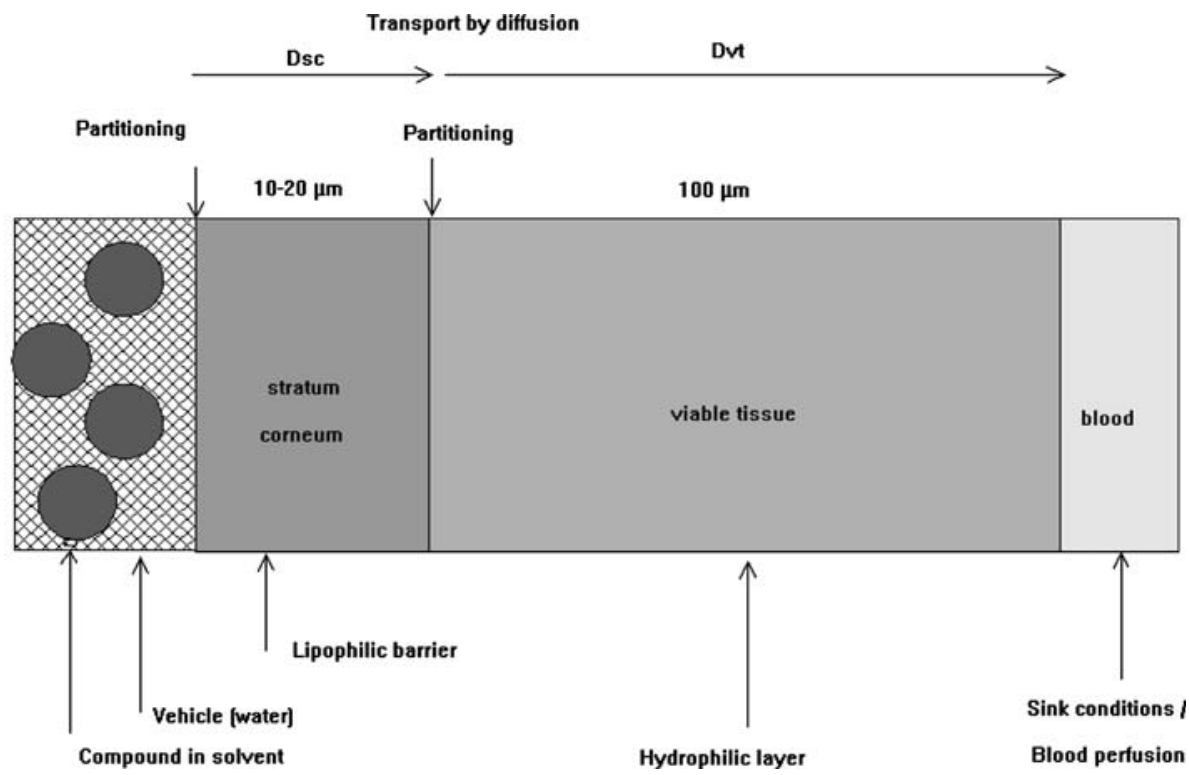


FORTRAN based Advanced Continuous Simulation Language (ACSL) software (Mitchell and Gauthier Associates, Cambridge, MA, USA). An important feature implemented in the model is the ability to simulate dermal absorptions during and after intermitting exposures.

In simulations of dermal permeation with the mechanistically based mathematical model usually the following assumptions are made with respect to the skin:

The thicknesses of the stratum corneum and the viable epidermis are $15 \mu \mathrm{m}$ and $100 \mu \mathrm{m}$, respectively (ECETOC 1993a). In the absence of a solution on the skin, the SC is covered with a thin water layer as assumed by McKone and Howd (1992). If solid material is applied on the skin (powder, dust, crystalline material), the concentration on the SC surface is limited by the solubility in the surface water layer. If the skin is in contact with a vapor, this will partition between the air and the thin water layer, and thereafter penetrate into the stratum corneum.

For the compound specific parameters in the model, such as the partition and diffusion coefficients, quantitative structure activity relations (QSARs) are used. If the penetrating compound is dissolved in water, the coefficients for partitioning between the $\mathrm{SC}$ and the water solution $\left(\mathrm{P}_{\mathrm{SC} / \mathrm{W}}\right)$ and between the $\mathrm{SC}$ and the viable epidermis $\left(\mathrm{P}_{\mathrm{SC} / \mathrm{VE}}\right)$ are assumed to be equal. They are derived from the octanolwater partition coefficient $\left(\mathrm{P}_{\mathrm{OW}}\right)$, according to Potts and Guy (1992) and Cleek and Bunge (1993). The diffusion coefficients in the SC and the VE are calculated according to the QSARs given by Potts and Guy (1992), Cleek and Bunge (1993) and Bunge and Cleek (1995), and are related to the molecular weight.

Thus, for a proper simulation of the dermal permeation the following compound specific information is required: the molecular weight (MW), the octanol-water partition coefficient $\left(\mathrm{P}_{\mathrm{OW}}\right)$, and in case of solid preparations deposited on the skin also the water solubility.

\subsubsection{Output of the simulation model}

The mathematical model enables the simulation of time courses of various quantities that play a role in the assessment of dermal absorption, such as:

- The penetration rate (flux through the skin into the body).

- The cumulative amount absorbed into the skin (and thus potentially absorbed into the body).

- The cumulative amount actually absorbed into the body (systemically absorbed).

- The actual amounts present on the skin (not yet absorbed) and in the skin.

Some of the features of the model are presented in the following two graphs (Figs. 4 and 5).

\subsubsection{Effects of formulation and application of plant protection products on the dermal absorption}

Various factors play a role in the dermal uptake of plant protection products. In this respect one may think of the use of dry (powder) or granular formulations, the limited solubility of the active ingredients, the solvent used in the stock solution or the formulation. Furthermore, the effects of dermal area dose (amount applied per skin area), the body surface exposed and the concentration in the solution on the skin may strongly affect the total amount absorbed via the skin. The average amount (daily) absorbed will also depend on the exposure pattern (single, repeated and multi-day exposures). The effects of these factors on the dermal uptake cannot be predicted in a simple way.

Mathematical modeling can serve as a helpful tool to simulate and assess these effects in a quantitative way.
Fig. 4 Dermal exposure during $8 \mathrm{~h}$ and an observation period of $24 \mathrm{~h}$. Simulation of the cumulative absorption into the skin (1), through the skin into the body (2), and amount actually present in the skin reservoir (3). Note that after cessation of the exposure $(t=8$ h) the systemic absorption will continue until all material has disappeared from the skin reservoir

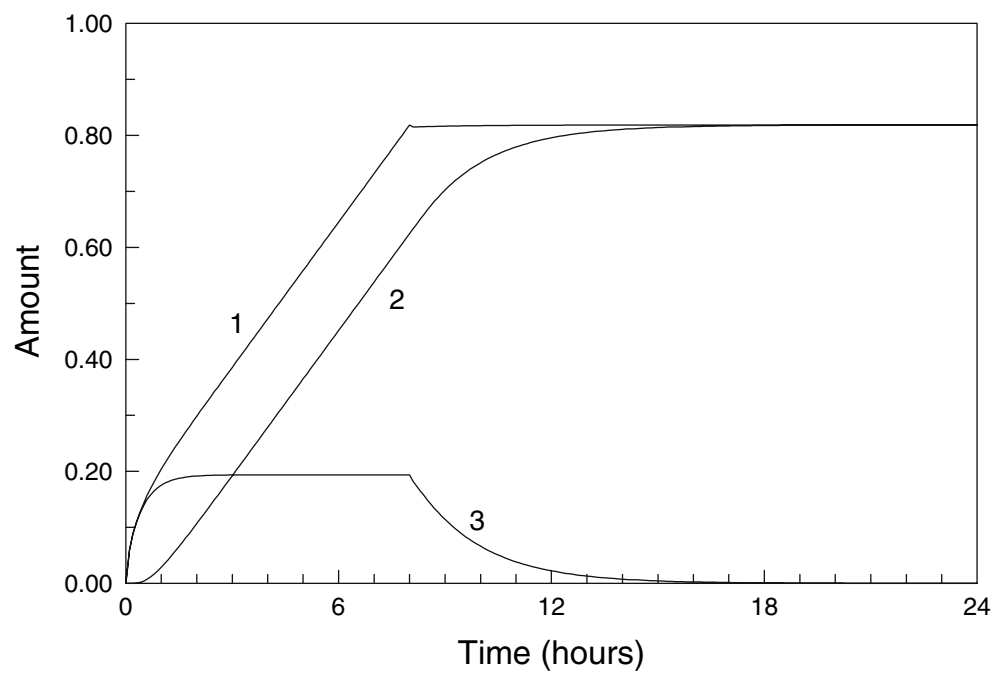


Fig. 5 Dermal exposure during $8 \mathrm{~h}$ and an observation period of $24 \mathrm{~h}$. Simulation of the absorption rate (1) and the cumulative absorption (2) through the skin into the body

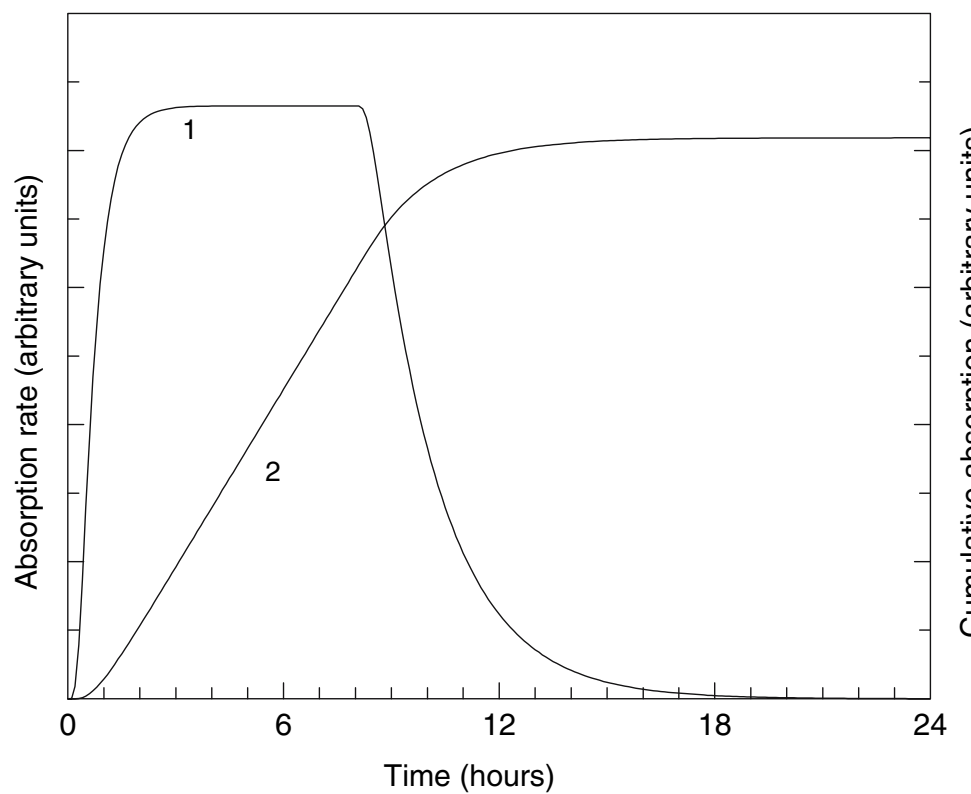

In the following three figures we will illustrate in which way the exposure of dry (powder), granular and nonaqueous (organic) solvent formulations, is implemented in the modeling of the dermal absorption (Figs. 6, 7, and 8).

The compound in the powder will first have to dissolve in the liquid (aqueous) surface layer on the skin before it may penetrate in to the skin and be absorbed. The absorption rate will be limited by the (water) solubility of the compound.

The compound is contained in the (mineral clay) granules. In this case the percentage of dermal absorption may be greatly reduced, when compared to the use of a liquid formulation, because of a reduced contact area between the granular formulation and the skin, and a limited rate of transport of the compound from the inside of the clay granules to the skin contact area.

Compared to the use of an aqueous solvent, the skin absorption will be affected in three ways. The concentration of the product in the vehicle may be higher due to increased solubility of the compound in the solvent (1). On the other hand the partition coefficient between the SC and the vehicle will be lower (2). The solvent may affect the barrier function of the skin, by irritation or dissolving skin component (3). The effect (1) and (3) will increase the dermal uptake, while (2) will reduce it.

In the following four graphs the effects of molecular weight and repeated exposure on the absorption rate and cumulative absorption will be illustrated. In the simulations the following scenarios have been used (Figs. 9, 10, 11, and 12).

- A single exposure during $8 \mathrm{~h}$ and simulation during $24 \mathrm{~h}$

- Repeated exposures during $8 \mathrm{~h}$ per days, for five consecutive days and simulation during 7 days.

An increase in molecular weight of the penetrating compound will result in a reduced value of the diffusion coefficient and a slower dermal permeation.
Fig. 6 A dry (powder) formulation deposited on the skin

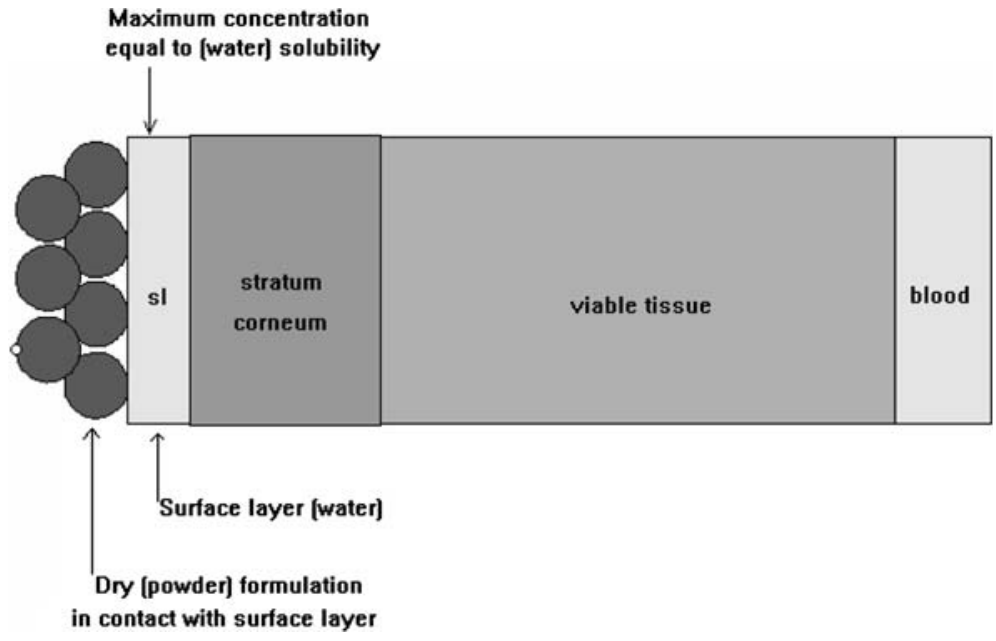


Fig. 7 Granular formulation deposited on the skin

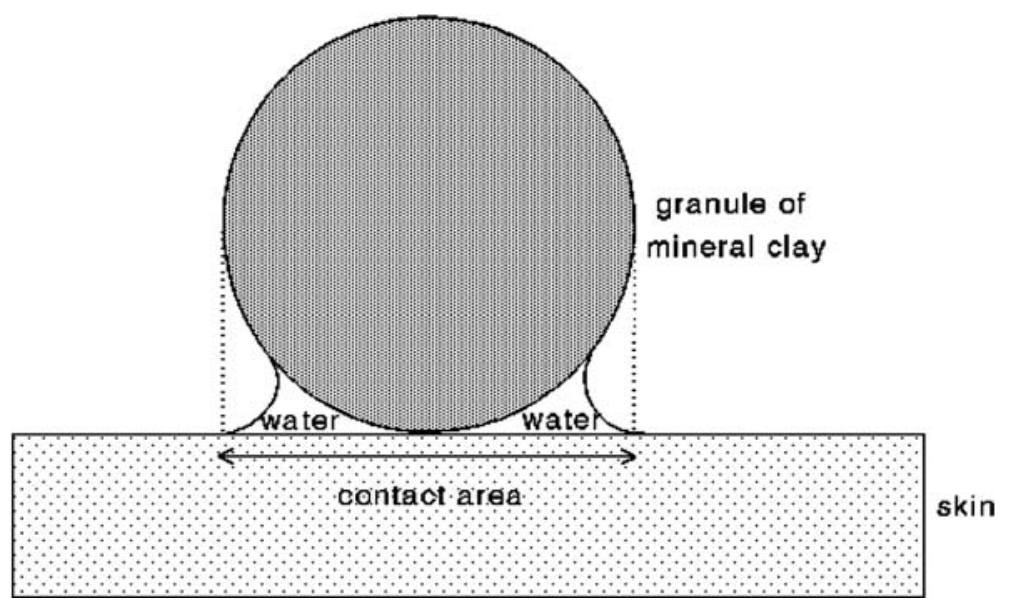

Fig. 8 Product dissolved in a non aqueous (organic) hydrophobic solvent

Fig. 9 Single dermal exposure during $8 \mathrm{~h}$ to a low molecular weight compound and an observation period of $24 \mathrm{~h}$. Simulation of the absorption rate (1) and the cumulative absorption (2) through the skin into the body. Note that the absorption is virtually completed after $18 \mathrm{~h}$ and definitely after $24 \mathrm{~h}$
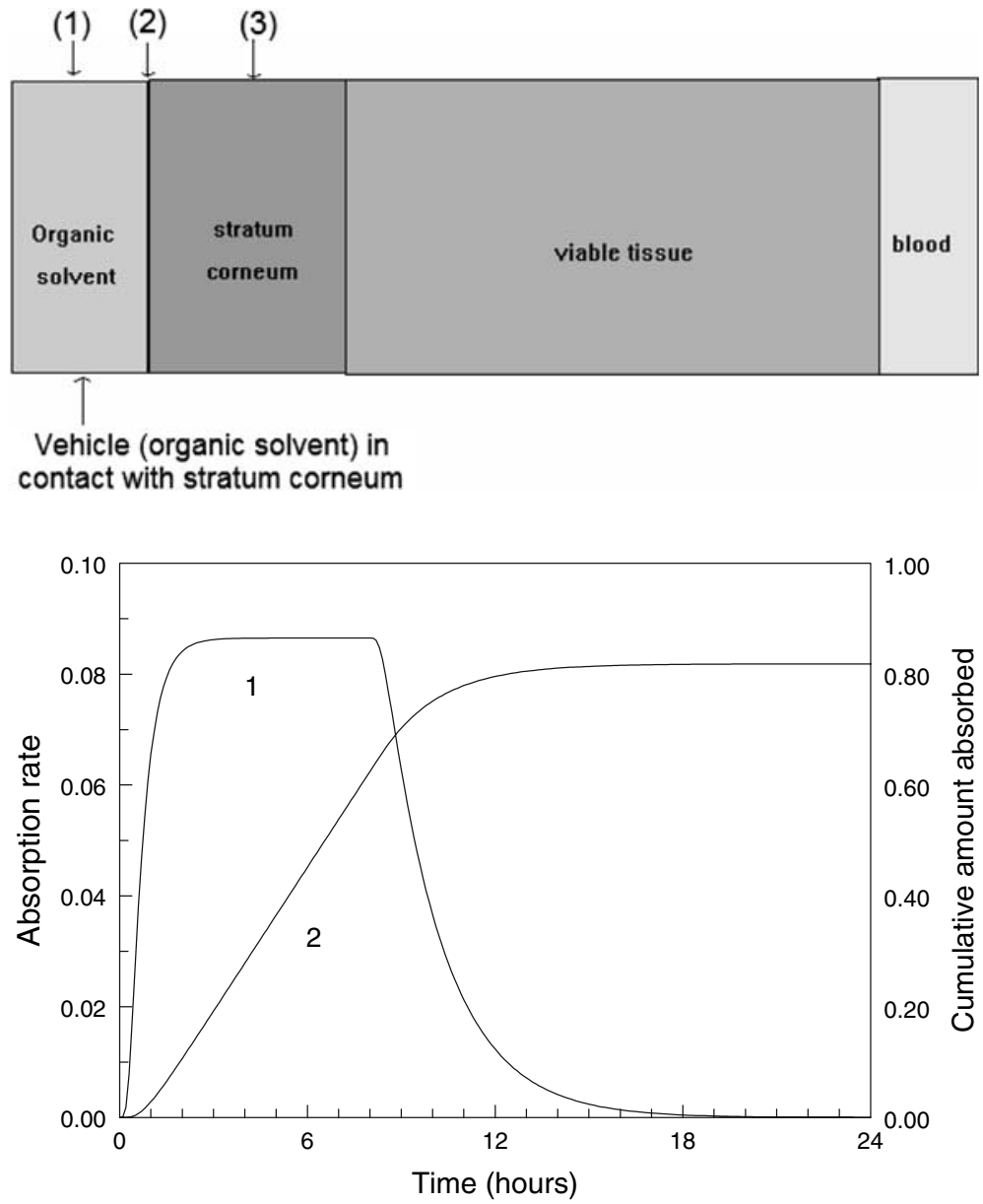

solution of certain volume and concentration is increased, while the total amount deposited on the skin remains the same, it will increase the total amount absorbed. In this case mathematical modeling can be a useful tool to estimate the effect of exposure area. For a proper dermal risk assessment information on the parts of the body that are exposed during the use of the pesticides should therefore be known. In some of the different dermal exposure models various skin areas are distinguished. 
Fig. 10 Repeated dermal exposure during $8 \mathrm{~h}$ per day for five consecutive days to a low molecular weight compound The dermal absorption is simulated during $168 \mathrm{~h}$ (one week). Simulation of the absorption rate (1) and the cumulative absorption body.Note that the absorption is not affected by the exposure on the previous day (2) through the skin into the

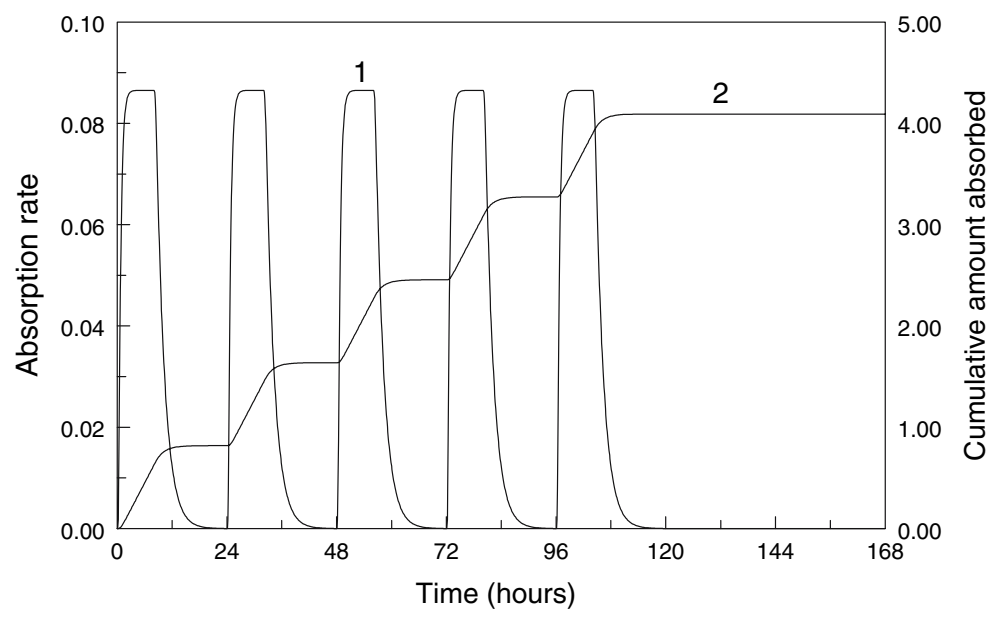

Fig. 11 Single dermal exposure during $8 \mathrm{~h}$ to a high molecular weight compound and an observation period of $24 \mathrm{~h}$. Simulation of the absorption rate (1) and the cumulative absorption (2) through the skin into the body. Note that the absorption is not completed after $24 \mathrm{~h}$

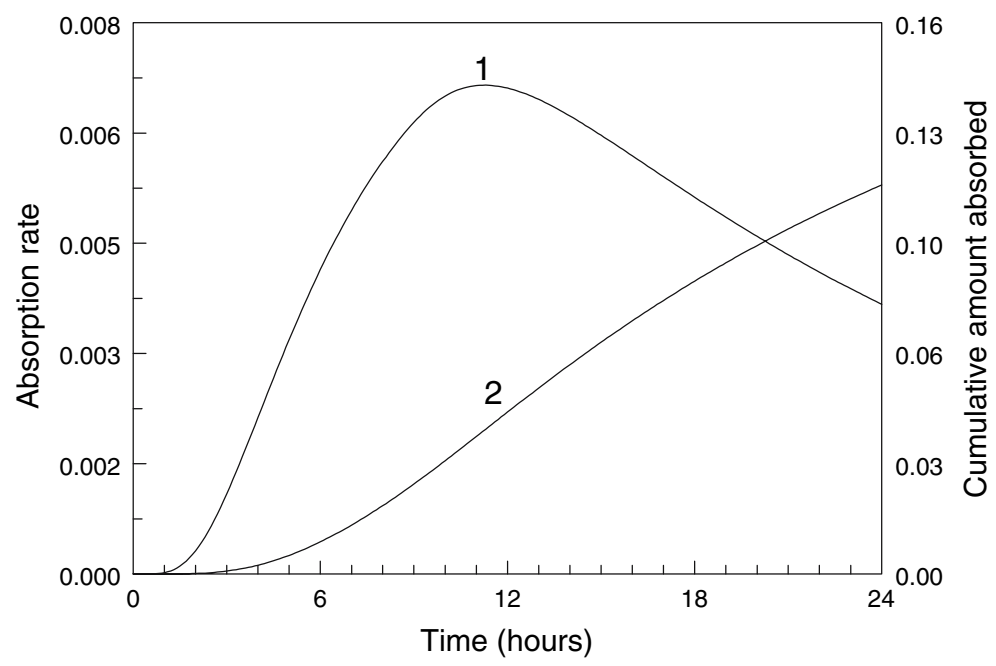

Fig. 12 Repeated dermal exposure during $8 \mathrm{~h}$ per day for five consecutive days to a high molecular weight compound. The dermal absorption is simulated during $168 \mathrm{~h}$ (one week).

Simulation of the absorption rate (1) and the cumulative absorption (2) through the skin into the body. Note that the absorption on the successive days is affected by the exposure on the previous days. The absorption is not fully completed at the end of the 7 day period, in spite of the absence of exposure at the last 2 days of the week

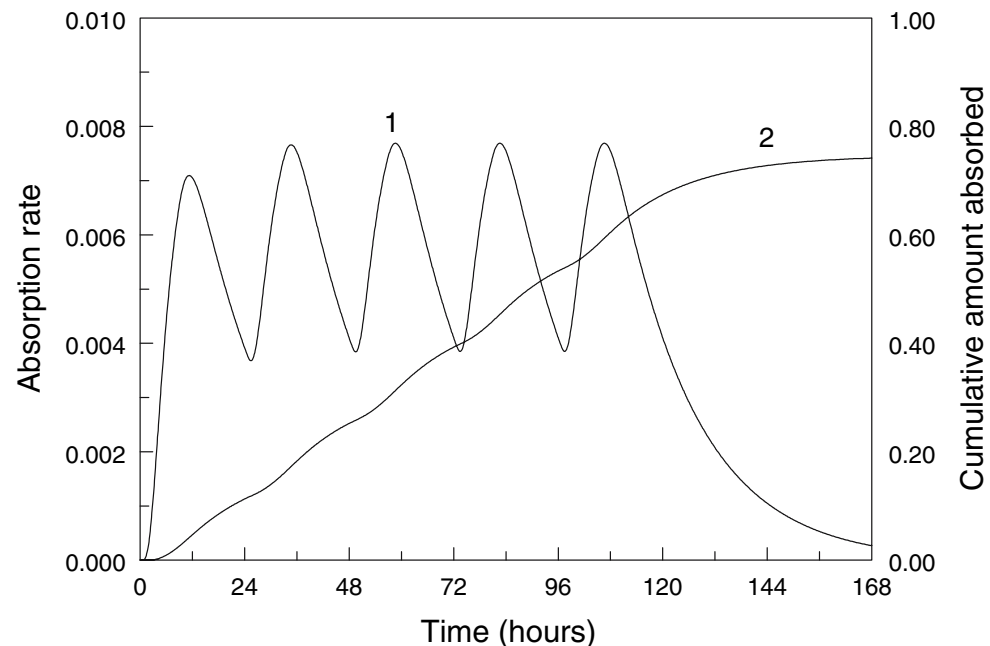

- German model (Lundehn et al. 1992)Hands, head and body

- UK model (POEM; PSD 1986 and 1992)Hands, trunk and legs

For other exposure models no specification of the exposed areas is given. In this case one must make assumptions on the exposure, e.g. exposure of hands and forearms during mixing/loading activities, and the whole body surface during application of the pesticide. Information on the surface areas of the whole body and the different body parts is given by Leung and Paustenbach (1994) and in ECETOC (1993b). 


\section{Discussion and conclusions}

In the mixing/loading and application of pesticides the skin is often the main route of systemic exposure of the workers. This is due to the form in which the pesticides are offered and handled and the way in which personal protective equipment (PPE) is used. In case of exposure to volatile substances and gases inhalation usually constitutes the single route of systemic uptake. The level of systemic exposure to these substances can usually be quantified in a straightforward way, whereby one may assume that the intake is proportional to the air concentration of the compound, the duration of exposure and the respiratory minute volume. For dermal exposure such a simple approach is not valid. Although various models and methods are available to estimate the external dermal exposure, it is not obvious to correlate this exposure to the level of systemic dermal uptake. Skin absorption is not only determined by the total amount deposited on the body, but also by the dermal area dose (amount per exposed skin area), the concentration in the solution, the volume deposited per skin area and the surface of the area exposed. Since dermal absorption is usually a relatively slowly proceeding process (especially in the case of higher molecular weight compounds), and because the skin acts as a reservoir (especially for lipophilic compounds), it will take some time before an equilibrium in the permeation rate (steady state) will be established. The non-steady state that will occur during short exposures will complicate the establishment of simple relationships between the duration of exposure and the amounts of dermal absorption.

Since, in contrast to exposure via inhalation, simple linear relationships between level and duration of external dermal exposure and systemic absorption cannot be established, a more detailed approach has to be followed.

In the mechanistically based mathematical model of dermal absorption, presented here, it is attempted to present a realistic description of the skin and the dermal absorption processes, without getting lost into too many details, that cannot be assessed in a quantitative way.

In this paper an illustration is given of some of the various features of this dermal absorption model. The model offers the possibility to integrate the different types of information that determine the dermal absorption, such as:

- Compound specific properties (molecular size, lipophilicity, solubility)

- The solvent or formulation used

- Exposure scenarios (single, repeated and multi-day)

- Exposure levels
In this way the model can serve as a useful tool in the occupational risk assessment of the use of pesticides. It may also be helpful in the development of new formulation and application techniques, not only aimed at an optimal efficacy of the pesticide, but also at an increase in the safety of usage.

\section{References}

AIR. (1996). The development, maintenance and dissemination of a European predictive operator exposure model (Europoem) database (Final draft report). Report nr. AIR3CT93-1370. A concerted action under the AIR (agriculture and agro-industry including fisheries) specific programme of the community's Third Framework Programme for Research and Technological development, and managed by DGVI.FII.3.

Bunge, A., \& Cleek, R. (1995). A new method for estimating dermal absorption from chemical exposure. 2: Effect of molecular weight and octanol-water partitioning. Pharmaceutical Research, 12, 88-95.

Cleek, R., \& Bunge, A., (1993). A new method for estimating dermal absorption from chemical exposure. 1: General approach. Pharmaceutical Research, 10, 497-506.

De Heer, C., Wilschut, A., Stevenson, H., \& Hakkert, B. C. (1999). Guidance document on the estimation of dermal absorption according to a tiered approach: an update. TNO Report V98 1237, 27 p, January 1999, Zeist, The Netherlands.

ECETOC. (1993a). Percutaneous absorption. Monograph 20.

ECETOC. (1993b). Strategy for assigning a "Skin Notation". Document 31.

Leung, H., \& Paustenbach, D. (1994). Estimating the percutaneous absorption of chemicals due to occupational and environmental exposure. Applied Occupational and Environmental Hygiene, 9, 187-197.

Lundehn, J. R., Westphal, D., Kieczka, D., Krebs, B., Löcher-Bolz, S., \& Maasfeld, W. (1992). Uniform principles for safeguarding the health of applicators of plant protection products (Uniform principles for operator protections); Mitteilungen aus der Biologischen Bundesanstalt für Land-und Forstwirschaft, Heft no. 277, Berlin, Germany.

McKone, T, \& Howd, R. A. (1992). Estimating dermal uptake of nonionic organic chemicals from water and soil. I: Unified fugacity-based models for risk assessment. Risk Analysis, 12, 543-557.

Potts, R., \& Guy, R. (1992). Predicting skin permeability. Pharmaceutical Research, 9, 663-669.

PSD. (1986). Scientific subcommittee on pesticides and British agrochemicals joint medical panel. Estimation of exposure and absorption of pesticides by spray operators, United Kingdom.

PSD. (1992). The predictive operator exposure model (POEM). A users guide, United Kingdom.

Van Golstein Brouwers, Y. G. C., Marquart, J., \& Van Hemmen, J. J. (1996). Assessment of occupational exposure to pesticides in agriculture. Part IV: Protocol for the use of generic exposure data. TNO report V96.120, The Netherlands.

Van Hemmen, J. J. (1992). Assessment of occupational exposure to pesticides in agriculture. Part I: General aspects. Part II: Mixing and loading. Part III: Application. S141-1/3. Ministry of Social Affairs and Employment (SZW), The Hague, The Netherlands. 